\title{
Perinatal lethal osteogenesis imperfecta
}

\author{
W G Cole, R Dalgleish
}

Abstract

Perinatal lethal osteogenesis imperfecta is the result of heterozygous mutations of the COL1A1 and COL1A2 genes that encode the $\alpha 1$ (I) and $\alpha 2(I)$ chains of type I collagen, respectively. Point mutations resulting in the substitution of Gly residues in Gly-X-Y amino acid triplets of the triple helical domain of the $\alpha 1$ (I) or $\alpha 2$ (I) chains are the most frequent mutations. They interrupt the repetitive Gly-X-Y structure that is mandatory for the formation of a stable triple helix. Most babies have their own private de novo mutation. However, the recurrence rate is about $7 \%$ owing to germline mosaicism in one parent.

The mutations act in a dominant negative manner as the mutant pro $\alpha$ chains are incorporated into type I procollagen molecules that also contain normal pro $\alpha$ chains. The abnormal molecules are poorly secreted, more susceptible to degradation, and impair the formation of the extracellular matrix. The collagen fibres

Department of Medical Genetics, University of Toronto and The Research Institute,

The Hospital for Sick Children,

555 University Avenue, Toronto, Ontario, M5G 1X8,

Canada

W G Cole

\section{Department of}

Genetics,

University of

Leicester,

Leicester LE1 7RH,

UK

R Dalgleish

Correspondence to Professor Cole.

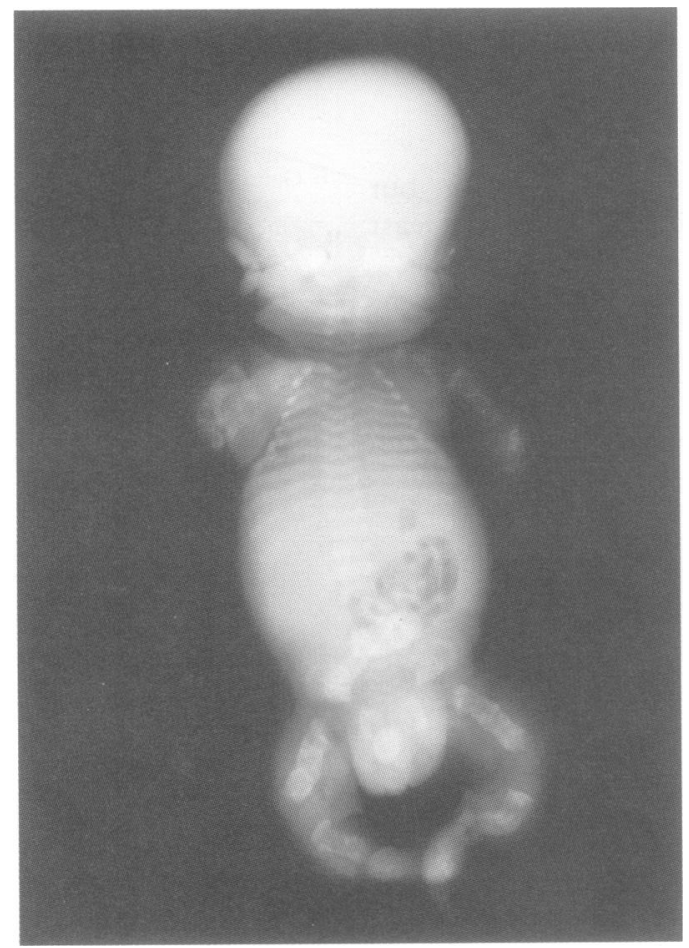

Figure 1 OI-ILA: radiograph showing severe osteopenia, continuously beaded ribs, platyspondyly, and broad crumpled long bones. are abnormally organised and mineralisation is impaired.

The severity of the clinical phenotype appears to be related to the type of mutation, its location in the $\alpha$ chain, the surrounding amino acid sequences, and the level of expression of the mutant allele.

\section{( $\mathcal{F}$ Med Genet 1995;32:284-289)}

Osteogenesis imperfecta (OI) is a genetically determined disorder of the connective tissues. The major phenotypic features include bone fragility, dentinogenesis imperfecta, blue sclerae, deafness, and ligamental laxity. It has long been recognised to be clinically heterogeneous with mild, moderate, severe, and lethal phenotypes. The Sillence classification takes these varying phenotypes into account. ${ }^{1}$

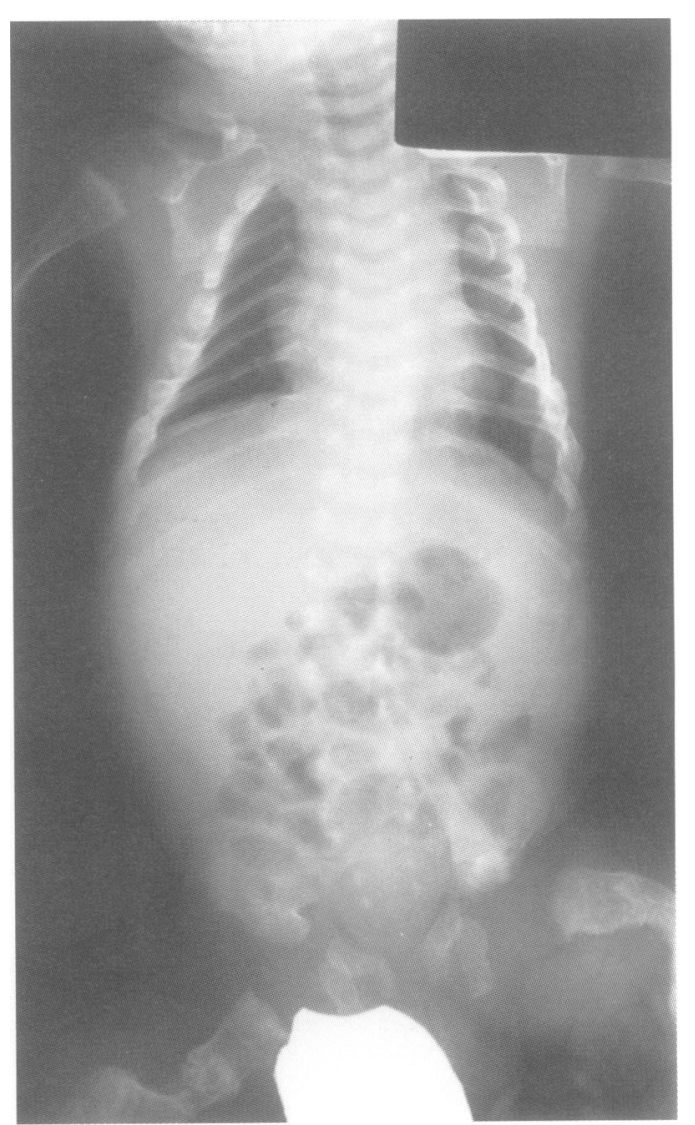

Figure 2 OI-IIB: radiograph showing severe osteopenia and broad crumpled femora. The ribs which are slender with expanded ends contain few fractures. 


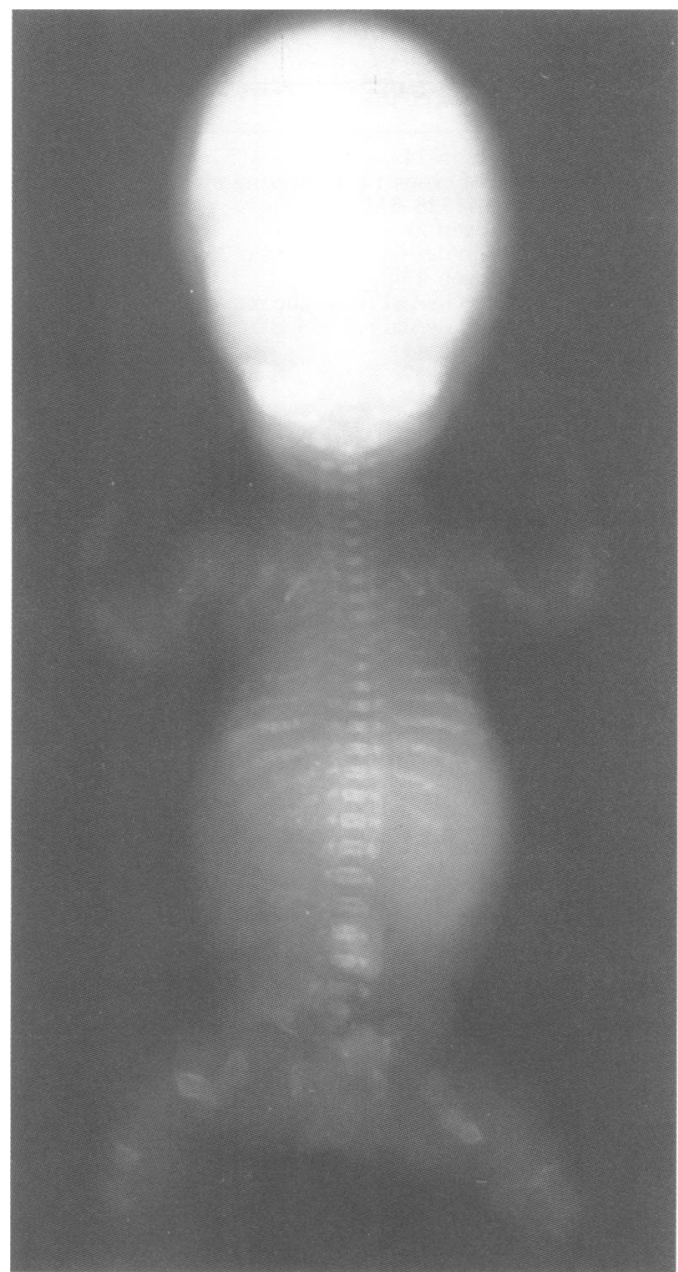

Figure 3 OI-IIC: radiography showing minimal ossification of the skeleton.

Type $I$ is the common mild form, type II is the perinatal lethal form, type III is a severe form, and type IV is a moderately severe form. We will focus on OI type II (OI-II).

\section{Clinical heterogeneity}

Babies are classified as having OI-II if they die in the perinatal period. However, the babies show clinical and radiological heterogeneity which appears to be established early in the pregnancy. ${ }^{2}$ The radiographic appearances are used to subclassify OI-II into groups A to C or into groups $\mathrm{I}$ to $\mathrm{V} .^{13}$ The Sillence subclassification will be used here.

\section{OI-IIA}

The babies are small for the period of gestation. The face is hypoplastic with micrognathia, deep blue-grey sclerae, and a relatively large calvarium, which is moulded and soft. The chest is small and the abdomen protuberant. The limbs are short and bowed. The hips are flexed and abducted. One or more limbs may be held in a severely deformed position. The radiographs show generalised osteopenia. The diaphyses of the long bones and ribs are as wide as their growth plates owing to the lack of metaphyseal remodelling (fig 1). The thin and crumpled cortices give the long bones a continuously beaded appearance. The pelvis and shoulder girdle have a similar appearance. There is generalised platyspondyly. The skull and face are severely porotic. Numerous wormian bones are present in the skull sutures. The babies are usually stillborn or die at birth.

OI-IIB

The ribs are less affected so that respiratory distress is less and babies may survive for months (fig 2). Otherwise the clinical and radiographic features are similar to those of OI-IIA.

\section{OI-IIC}

This is the least common subtype. The babies are very small, osteopenia is severe, and the long bones are slender, poorly modelled, and contain numerous fractures (fig 3). The babies are stillborn or die soon after birth.

\section{Genetic heterogeneity}

Numerous studies have shown that the majority

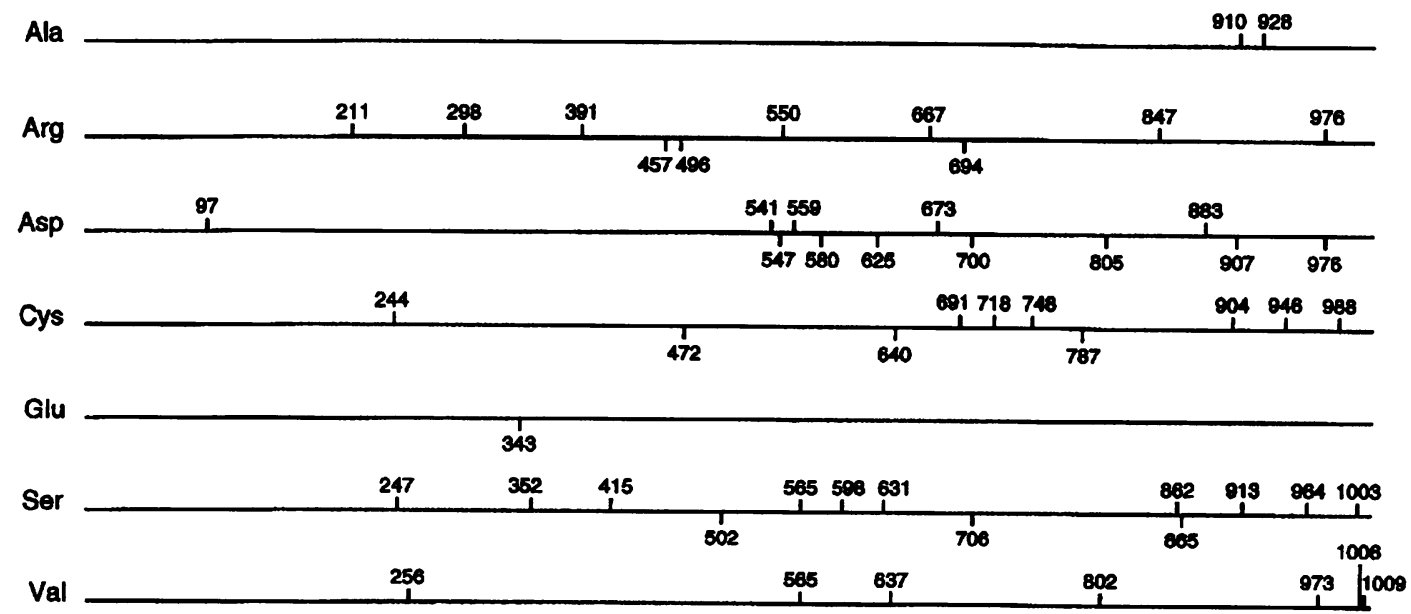

Figure 4 Map of glycine substitutions within the triple helical domains of the $\alpha 1(I)$ and $\alpha 2(I)$ chains of OI-II. Each line represents the triple helical domain of an $\alpha$ chain. The substituting residue is listed on the left. The residue numbers are indicated above each line for the $\alpha 1$ (I) chain and below each line for the $\alpha 2(I)$ chain. The residues are numbered relative to the first glycine residue of the triple helix. 
Exon skipping mutations, deletions, and insertions of the triple helical domains of COL1A1 and COL1A2 and the carboxyl-terminal propeptide domain of COL1A1 in OI-II

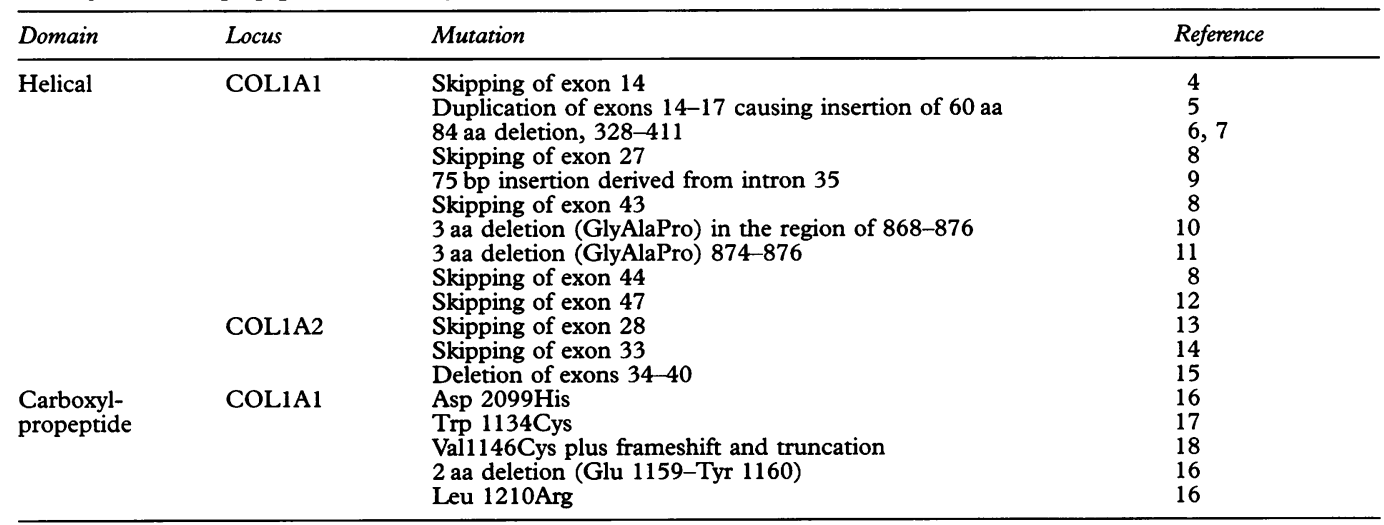

of babies with OI-II are heterozygous for mutations of the COL1A1 or COL1A2 genes that alter the structure of the triple helical domains or the carboxyl-terminal propeptides of the proa1(I) or proa2(I) chains of type I procollagen (fig 4, table). Each procollagen molecule contains two pro $\alpha 1$ (I) chains and one proa2(I) chain. The propeptides are enzymatically removed outside the cell to produce type I collagen that is laid down in the extracellular matrix of the dermis, bone, dentine, sclera, ligaments, and fascia, the tissues affected in OI.

The first mutation defined in OI-II was a heterozygous deletion within COL1A1 that resulted in the loss of 84 amino acids from the triple helical domain of the $\alpha 1$ (I) chain. ${ }^{67}$ However, few deletions have subsequently been identified (table). Exon skipping mutations are more common. They usually maintain the translational reading frame and the repetitive Gly-X-Y amino acid triplet structure as the exons encode complete triplets (table).

The majority of mutations in OI-II involve the substitution of Gly residues in Gly-X-Y triplets within the triple helical domain of the $\alpha$ chains. The ability of such mutations to produce the OI-II phenotype was confirmed by reproducing it in transgenic mice bearing a COL1A1 mutation that substituted Cys for Gly $859 .{ }^{19}$ Numerous point mutations of the GGN codon for Gly have been identified (fig 4). Substitutions by Ser are the most common while substitutions by Ala and Glu are rare and substitutions by Trp have not been identified to date. Approximately two-thirds of the substitutions involve the $\alpha 1(\mathrm{I})$ chain and a similar proportion involve the carboxyl-terminal halves of the triple helical domains of the $\alpha 1$ (I) and $\alpha 2(\mathrm{I})$ chains. The non-uniform nature of the substitutions, $\alpha$ chain involved, and sites within the $\alpha$ chains are unlikely to be the result of ascertainment or technical biases. Similar results were found when all babies born with OIII in Victoria, Australia were studied. ${ }^{17}$ Similar results have also been obtained with modern methods of mutation detection that screen full length $\alpha 1(\mathrm{I})$ and $\alpha 2(\mathrm{I})$ cDNAs. $^{20}$

Most unrelated babies with OI-II have their own private de novo mutation (fig 4). Exceptions include recurrent substitutions of Gly
352,415 , and 1003 by Ser in the $\alpha 1(\mathrm{I})$ chain. ${ }^{20-24}$ These mutations occurred at $\mathrm{CpG}$ dinucleotides, in a manner consistent with deamination of a methylated cytosine residue. ${ }^{21}$ However, there do not appear to be any major mutational hot spots in the COL1A1 or COL1A2 genes in babies with OI-II.

A few mutations in the carboxyl-propeptide of proal(I) chains have been identified in OIII (table). They alter sequences that are important in chain association, disulphide linking of the chains, and interactions with chaperones. ${ }^{1618}$

In many instances, the mutations in OIII are new dominant mutations, while in the remainder the mutation is inherited from a mosaic parent. ${ }^{25}$ Isolated germline mosaicism has not been identified to date. ${ }^{2526}$ The mosaic parent may appear to be clinically normal or to have a mild or moderate form of OI. However, leucocytes, hair follicles, and dermal fibroblasts contain differing proportions of the normal and mutant alleles. ${ }^{2526}$ The proportion within the osteoblasts, which is likely to be the most important determinant of the phenotype, is usually unknown. The recurrence risk of OIII is approximately $7 \%$ because of the occurrence of germline mosaicism in one parent. ${ }^{27}$

\section{Pathogenesis}

All of the mutations that produce OI-II appear to act in a dominant negative manner. The mutant pro $\alpha$ chains are incorporated into type I procollagen molecules and produce major abnormalities in collagen metabolism.

Deletions, skipping mutations, and insertions in the triple helical domain produce abnormal alignment of the pro $\alpha$ chains. Substitutions of Gly residues interrupt the mandatory repetitive Gly-X-Y structure required for normal formation of the triple helix. They may also produce a bulge or kink as a means of accommodating the bulkier side chains. For example, kinks at the mutant site have been observed in type I collagen molecules containing mutant $\alpha 1(\mathrm{I})$ chains bearing substitutions of Gly 718 or 748 by Cys. ${ }^{2829}$ The lysine residues, particularly on the amino-terminal side of the substitution, are often overmodified by the prolonged action of lysyl 
hydroxylase and glycosyl transferases owing to a delay in the zipper-like folding of the collagen molecules. ${ }^{30}$ The thermal denaturation temperature of the abnormal collagen is often reduced but it is independent of the level of overmodification of the $\alpha$ chains. ${ }^{31}$ The variable levels of overmodification and thermal stability of the mutant molecules are likely to reflect regional differences in helix stability and in the propensity for renucleation of folding of the helix beyond the mutation. ${ }^{32-34}$

As type I collagen molecules contain two $\alpha 1$ (I) chains it is likely with heterozygous mutations of COL1A1 that $25 \%$ of the molecules are normal while $75 \%$ of the molecules contain one or two mutant $\alpha 1$ (I) chains. Conversely, as type I collagen molecules contain one $\alpha 2(\mathrm{I})$ chain, it is likely with heterozygous mutations of COL1A2 that $50 \%$ of the molecules are normal and $50 \%$ contain a mutant $\alpha 2(\mathrm{I})$ chain. ${ }^{27}$ Procollagen containing one or more mutant pro $\alpha$ chains is poorly secreted and is excessively degraded by cultured fibroblasts. ${ }^{22}$ Once secreted, cleavage of the amino propeptides by $\mathrm{N}$-proteinase may be reduced even with mutations distant from the cleavage site. ${ }^{28}$

Histological and biochemical anomalies have been observed in the dermis from babies with OI-II. The fibroblasts often contain dilated, rough endoplasmic reticulum because of impaired secretion of the mutant type I procollagen molecules. ${ }^{35}$ The collagen fibrils are smaller than normal. There is a reduced amount of type I collagen which consists of a mixture of normal and mutant type I collagen molecules. The mutant collagen chains are more readily extracted which suggests that the type I collagen molecules containing one or more mutant chains may be degraded before they are incorporated into the more insoluble cross linked extracellular matrix. ${ }^{36}$

Severe anomalies have also been observed in bone from babies with OI-II. The bone is severely porotic. The normal cortical and trabecular bone pattern is replaced by woven bone. ${ }^{35} 37$ There is an abundance of plump osteoblasts surrounded by little extracellular matrix. The osteoblasts may also contain dilated rough endoplasmic reticulum. ${ }^{2}$ The growth plate is normal but cartilage cores persist in the trabeculae. ${ }^{38}$ The bone matrix contains a reduced amount of type I collagen and increased amounts of type III and V collagens. ${ }^{3639}$ The type I collagen includes both normal and mutant molecules. ${ }^{3640}$ The levels of some non-collagenous proteins are also abnormal. ${ }^{41}$ Osteocalcin, $\alpha 2-H S$ glucoprotein, and bone sialoprotein levels are increased, osteonectin is reduced, and decorin levels are normal. There are dramatic changes in the collagen fibrils and mineralisation. The collagen fibrils are thin and do not show the normal spatial arrangement of parallel bundles. ${ }^{22}$ At the mineralising front, apatite crystallites are not oriented along the collagen fibrils as observed in normal bone. The crystallites are also small and appear to grow by aggregation of new crystallites onto their surfaces. ${ }^{23843}$

Many of these metabolic findings were also observed in transgenic mouse models of
OI-II. ${ }^{1944}$ Expression of COL1A1 constructs encoding a Gly 859 to Cys substitution or an in frame deletion in the triple helical domain of $\alpha 1$ (I) chains resulted in the incorporation of mutant type I collagen molecules into the tissues.

Mutations of the carboxyl-terminus of the proa1(I) chain can also produce dramatic changes in type I collagen metabolism. ${ }^{1645}$ In one heterozygous baby with OI-II, a codon frameshift mutation produced a truncated pro $\alpha 1(\mathrm{I})$ chain with other pro $\alpha$ chains. ${ }^{18}$ The abnormal type I procollagen molecules were not secreted and were degraded within the cell. The type I collagen content of the dermis and bone was reduced to about $20 \%$ of normal. This is the amount of normal collagen that would be expected if all of the molecules containing one or two mutant proal(I) chains were degraded. In contrast, mutations of the carboxyl-terminus of the pro $\alpha 1(\mathrm{I})$ chains that prevent chain association and incorporation of mutant chains into type I procollagen molecules produce one form of mild OI-I. ${ }^{46}$

\section{Genotype-phenotype relationships}

There are two broad categories of genotypephenotype relationships in osteogenesis imperfecta. The first category includes phenotypes associated with haploinsufficiency of COL1A1 and COL1A2. The second category includes phenotypes associated with dominant negative mutations of COL1Al and COL1A2. There are rare exceptions, such as some forms of autosomal recessive OI that do not appear to be the result of type I collagen mutations. ${ }^{47}$

Haploinsufficiency of COL1A1 results in an approximately $50 \%$ reduction of type I collagen synthesis and the mild osteogenesis imperfecta type I phenotype with normal teeth, OI-IA. ${ }^{27}$ Heterozygous Mov-13 mice, in which one COL1A1 allele is inactivated by insertion of a retrovirus, also show the OI-IA phenotype. ${ }^{48}$ In contrast, homozygous Mov-13 mice die around the twelfth day of gestation as a result of the absence of type I collagen from many tissues. ${ }^{49}$ A human equivalent has not been identified as yet but would probably produce early death in utero and spontaneous abortion.

A homozygous deficiency of $\alpha 2$ (I) chains has been reported in a child with moderately severe OI-III. ${ }^{50}$ The structure of the carboxyl-propeptide of the proa2(I) chains was altered so that the chains would not associate. The metabolic consequences of the mutation were equivalent to homozygous haploinsufficiency of COL1A2. The heterozygous parents were minimally affected.

The majority of deletions, insertions, and splicing anomalies that alter the triple helical domain of the $\alpha 1(\mathrm{I})$ or $\alpha 2(\mathrm{I})$ chains produce lethal phenotypes. However, the more common glycine substitutions in the repetitive Gly-X-Y triplets of the triple helix produce mild to lethal phenotypes. The site and type of substitution have been proposed as important factors in determining the severity of the phenotype. For Gly substitutions by 
Cys in the $\alpha 1$ (I) chain, carboxyl-terminal sites produce lethal phenotypes, mid-helical sites produce moderately severe phenotypes, and amino-terminal sites produce mild phenotypes. $^{34}$ However, exceptions such as the substitution at Gly 244 by Cys in a baby with OI-II have been observed. ${ }^{51}$ Gradients of phenotypic severity are less obvious with other Gly substitutions (fig 4). Lethal phenotypes are frequently observed with mid-helical Gly substitutions by Ser and Arg.

There are a few rare instances of unrelated subjects harbouring the same substitutions of glycines in either the $\alpha 1(\mathrm{I})$ or $\alpha 2(\mathrm{I})$ chain. Somewhat unexpectedly, such people do not always exhibit the same phenotype suggesting the possible involvement of epistatic effects. To illustrate this, an $\alpha 1$ (I)-Gly352Ser substitution produces OI-II in one person but OI-IV in two others. ${ }^{20223} \mathrm{~A}$ similar, though less dramatic, effect has also been noted in two subjects with OI-III resulting from an $\alpha 2(\mathrm{I})-\mathrm{Gly} 859$ Ser substitution. ${ }^{52}$ In contrast, three subjects harbouring an $\alpha 2(\mathrm{I})-\mathrm{Gly} 502 \mathrm{Ser}$ substitution appear to exhibit exactly the same OI-II phenotype. ${ }^{53}$ Clearly evidence from more instances is required to resolve the question of epistasis

Gly substitutions were proposed to be less deleterious in the $\alpha 2(\mathrm{I})$ chain as type I collagen molecules only contain one $\alpha 2(\mathrm{I})$ chain. However, a similar proportion of Gly substitutions in the $\alpha 1(\mathrm{I})$ and $\alpha 2(\mathrm{I})$ chains produce lethal phenotypes. The sequences around the Gly substitution may be more important in determining the consequences of the substitution than the $\alpha$ chain involved. ${ }^{23}{ }^{32}$ For example, within the $\alpha 2(\mathrm{I})$ chain there are non-lethal domains interspersed wth lethal domains. ${ }^{23}$

The level of expression of the mutant allele has been shown in transgenic mice also to be an important determinant of the severity of the phenotype. A direct relationship was found between the severity of the phenotype and steady state mutant mRNA levels in mice bearing a Gly 859 substitution by Cys in COL1A1. ${ }^{19}$ These findings indicated that moderate levels of expression of the mutant COL1A1 were sufficient to lead to dramatic dominant negative effects on the incorporation of type I collagen into the extracellular matrix. Similar findings were also observed in transgenic mice that were high expressors of a COL1A1 gene construct containing an in frame deletion. ${ }^{44}$ However, inbred mice expressing moderate levels of this transgene showed marked phenotypic variability. ${ }^{54}$ The explanation for this variability was not determined.

Little is known about the levels of expression of the mutant alleles and the levels of incorporation of mutant molecules into the extracellular matrix in human OI-II. However, the collagen content of the dermis and bone is reduced and mutant collagen is detectable in babies with Gly substitutions in the triple helical domain of the $\alpha$ chains. ${ }^{36}$ In one baby with a substitution of Gly 580 by Asp in the $\alpha 2(\mathrm{I})$ chain, the bone matrix was shown to contain a ratio of mutant to normal $\alpha 2(\mathrm{I})$ chains of $0 \cdot 7: 1$. $^{40}$

The genotypes associated with subtypes of
OI-II have only been studied in a few babies. The substitution of Gly by Arg at Gly 391 produced OI-IIA, at Gly 667 produced OIIIA/IIB, and at Gly 976 produced OI-IIB. ${ }^{35}$ The substitution of Gly by Val at Gly 1006 produced OI-IIC, Gly 973 produced OI-IIA, and Gly 256 produced IIA. ${ }^{55}$ A carboxyl-propeptide mutation of the proal(I) chain produced OI-IIB. ${ }^{45}$

\section{Prenatal diagnosis}

OI-II is often detected on routine ultrasound scans of pregnant women with a history of osteogenesis imperfecta. ${ }^{567}$ The skull is poorly echogenic and the limb bones are characteristically broad, shortened, and fractured. The chest is also abnormal. OI-IIA is the most common subtype. ${ }^{5658}$ These scans are often undertaken at about 16 weeks but can be done earlier in families with a history of previous babies with OI-II. In the latter families, intrauterine detection of the COL1A1 or COL1A2 mutations can be used if the mutation has been determined before the pregnancy.

Some of this work was supported with grants from the Medica Research Council of Canada (WGC) and the Medical Research Council (RD)

1 Sillence DO, Senn A, Danks DM. Genetic heterogeneity in

2 Cohen-Solal L, Zylberberg L, Sangalli A, Gomez Lira M Mottes M. Substitution of an aspartic acid for glycine 700 in the $\alpha 2$ (I) chain of type I collagen in a recurrent lethal type II osteogenesis imperfecta dramatically affects th mineralization of bone. $\mathcal{F}$ Biol Chem 1994;269:14751-8.

3 Byers PH, Tsipouras P, Bonadio JF, Starman BJ, Schwartz RC. Perinatal lethal osteogenesis imperfecta (OI type II) a biochemically heterogeneous disorder usually due to new mutations in the genes for type I collagen. Am $\mathcal{f}$ Hum Genet 1988;42:237-48.

4 Bonadio J, Ramirez F, Barr M. An intron mutation in the human $\alpha 1(\mathrm{I})$ collagen gene alters the efficiency of premRNA splicing and is associated with osteogenesis im perfecta type II 7 Biol Chem 1990:265.2262-8.

5 Cohn DH, Zhang X, Byers PH. Homology-mediated recombination between type I collagen gene exons results in an internal tandem duplication and lethal osteogenesis in an internal tandem duplication and

6 Chu M-L, Gargiulo V, Williams CJ, Ramirez F. Multiexon Chu M-L, Gargiulo V, Williams CJ, Ramirez F. Multiexon deletion in an osteogenesis imperfecta variant with increased $691-4$.

7 Barsh GS, Roush CL, Bonadio J, Byers PH, Gelinas RE. Intron-mediated recombination may cause a deletion of an $\alpha 1$ type I collagen chain in a lethal form of osteogenesis imperfecta. Proc Natl Acad Sci USA 1985;82:2870-4.

8 Byers PH. Brittle bones - fragile molecules: disorders of collagen gene structure and expression. Trends Genet 1990 6:293-300.

9 Genovese C, Brufsky A, Shapiro J, Rowe D. Detection of mutations in human type I collagen mRNA in osteogenesis imperfecta by indirect RNase protection. $7 \mathrm{Biol}$ Chem 1989;264:9632-7.

10 Hawkins JR, Superti-Furga A, Steinmann B, Dalgleish R A 9-base pair deletion in COL1A1 in a lethal variant of
osteogenesis imperfecta. $\mathcal{F}$ Biol Chem 1991;266:22370-4.

11 Wallis GA, Kadler KE, Starman BJ, Byers PH. A tripeptide deletion in the triple-helical domain of the pro $\alpha 1$ (I) chain of type I procollagen in a patient with lethal osteogenesis of type I procollagen in a patient with lethal osteogenesis imperfecta does not alter cleavage of the mol

12 Wallis GA, Starman BJ, Chessler SD, Willing MC, Byer PH. Mutations in the CB6 and carboxyl-terminal regions PH. Mutations in the CB6 and carboxyl-terminal regions of COL1A1 causing lethal OI have different effects on the stability and secretion of the type I collagen molecule. IV International Conference on Osteogenesis

13 Tromp G, Prockop DJ. Single base mutation in the pro $\alpha 2$ (I) collagen gene that causes efficient splicing of RNA from exon 27 to exon 29 and synthesis of a shortened but in frame pro $\alpha 2(\mathrm{I})$ chain. Proc Natl Acad Sci USA 1988;85: $5254-8$

14 Ganguly A, Baldwin CT, Strobel D, Conway D, Horton W Prockop DJ. Heterozygous mutation in the $\mathrm{G}^{+5}$ position of intron 33 of the pro- $\alpha 2$ (I) gene (COL1A2) that causes aberrant RNA splicing and lethal osteogenesis imperfecta: use of carbodiimide methods that decrease the extent of DNA sequencing necessary to define an unusual mutation. f Biol Chem 1991;266:12035-40.

15 Willing MC, Cohn DH, Starman B, Holbrook KA, Green- 
berg CR, Byers PH. Heterozygosity for a large deletion in the $\alpha 2(\mathrm{I})$ collagen gene has a dramatic effect on type collagen secretion and produces perinatal lethal osteogenesis imperfecta. F Biol Chem 1988;263:8398-404

16 Chessler SD, Wallis GA, Byers PH. Mutations in the carboxyl-terminal propeptide of the pro $\alpha 1$ (I) chain of type I collagen result in defective chain association and produce lethal osteogenesis imperfecta. F Biol Chem 1993;268: 18218-25.

17 Bateman JF, Lamande SR, Hannagan M. Moeller I, Dah HHM, Cole WG. Chemical cleavage method for the detection of RNA bases changes: experience in the application to collagen mutations in osteogenesis imperfecta. Am $\mathcal{F}$ Med Genet 1993;45:233-40.

18 Bateman JF, Lamande SR, Dahl HHM, Chan D, Mascara T, Cole WG. A frameshift mutation results in a truncated nonfunctional carboxyl-terminal pro $\alpha 1(\mathrm{I})$ propeptide of nonfunctional carboxyl-terminal proal(I) propeptide of type I collagen in

19 Stacey A, Bateman J, Choi T, Mascara T, Cole W, Jaenisch R. Perinatal lethal osteogenesis imperfecta in transgenic mice bearing an engineered mutant pro- $\alpha 1$ (I) collagen gene. Nature 1988;332:131-6.

20 Mackay K, Byers PH, Dalgleish R. An RT-PCR-SSCP screening strategy for detection of mutations in the gen encoding the $\alpha 1$ chain of type I collagen: application to four patients with osteogenesis imperfecta. Hum Mol Genet 1993;2:1155-60.

21 Pruchno CJ, Cohn DH, Wallis GA, et al. Osteogenesis imperfecta due to recurrent point mutations at $\mathrm{CpG}$ dinucleotides in the COL1A1 gene of type I collagen. Hum Genet 1991;87:33-40.

22 Bateman JF, Moeller I, Hannagan M, Chan D, Cole WG Characterization of three osteogenesis imperfecta collagen Characterization of three osteogenesis imperfecta collagen $\alpha 1$ (I) glycine to serine mutations demonstrating a position288:131-5.

23 Marini C, Lewis MB, Wang Q, Chen KJ, Orrison BM Serine for glycine substitutions in type I collagen in two cases of type IV osteogenesis imperfecta (OI). Additional evidence for a regional model of OI pathophysiology. $f$ Biol Chem 1993;268:2667-73.

24 Mottes M, Gomez Lira MM, Valli M, et al. Paternal mosaicism for a COL1A1 dominant mutation ( $\alpha 1$ Ser-415) causes recurrent osteogenesis imperfecta. Hum Mutat 1993;2:196-204.

25 Wallis GA, Starman BJ, Zinn AB, Byers PH. Variable expression of osteogenesis imperfecta in a nuclear family is in the $\alpha 1$ (I) gene (COL1A1) of type I collagen in a parent. Am f Hum Genet 1990;46:1034-40.

26 Cohn DH, Starman BJ, Blumberg B, Byers PH. Recurrence of lethal osteogenesis imperfecta due to parental mosaicism for a dominant mutation in a human type I collagen gen (COL1A1). Am $¥$ Hum Genet 1990;46:591-601.

27 Byers PH, Wallis GA, Willing MC. Osteogenesis imperfecta: translation of mutation to phenotype. $\mathscr{f}$ Med Genet 1991 28:433-42.

28 Lightfoot SJ, Holmes DF, Brass A, Grant ME, Byers PH, Kadler KE. Type I procollagens containing substitutions of aspartate, arginine and cysteine for glycine in the proal(I) chain are cleaved slowly by $N$-proteinase, but only the cysteine substitution introduces a kink into the molecule. f Biol Chem 1992;267:25521-8.

29 Vogel BE, Doelz R, Kadler KE, Hojima Y, Engel J, Prockop DJ. A substitution of systeine for glycine 748 of the $\alpha 1$ chain produces a kink at this site in the procollagen I molecule and an altered $N$-proteinase cleavage site ove $225 \mathrm{~nm}$ away. F Biol Chem 1988;263:19249-55.

30 Raghunath M, Bruckner P, Steinmann B. Delayed triple helix formation of mutant collagen from patients with osteogenesis imperfecta. F Mol Biol 1994;236:940-9.

31 Rao VH, Steinmann B, de Wet W, Hollister DW. Decreased thermal denaturation temperature of osteogenesis imperfecta mutant collagen is independent of post-transperfecta mutant collagen is independent of post-transBiol Chem 1989;264:1793-8.

32 Bächinger HP, Morris NP, Davis JM. Thermal stability and folding of the collagen triple helix and the effects of mutations in osteogenesis imperfecta on type I collagen Am $\mathcal{F}$ Med Genet 1993;45:152-62.

33 Westerhausen A, Kishi J, Prockop DJ. Mutations that substitute serine for glycine $\alpha 1-598$ and glycine $\alpha 1-631$ in type I procollagen. The effects on thermal unfolding of the triple helix are position-specific and demonstrate that the protein unfolds through a series of cooperative blocks. f Biol Chem 1990;265:13995-4000.

34 Wenstrup RJ, Shrago-Howe AW, Lever LW, Phillips CL, Byers PH, Cohn DH. The effects of different cysteine for glycine substitutions within $\alpha 2(\mathrm{I})$ chains. Evidence of distinct structural domains within the type I collagen triple helix. F Biol Chem 1991;266:2590-4.

35 Cole WG, Chow CW, Rogers JG, Bateman JF. The clinical features of three babies with osteogenesis imperfecta resulting from the substitution of glycine by arginine in the pro $\alpha 1$ (I) chain of type I procollagen. $\mathcal{F}$ Med Genet 1990 27:228-35.

36 Bateman JF, Chan D, Mascara T, Rogers JG, Cole WG. Collagen defects in lethal perinatal osteogenesis imperfecta. Biochem $\mathcal{F}$ 1986;240:699-708.

37 Sztrolovics R, Glorieux FH, Travers R, van der Rest $M$ Roughly PJ. Osteogenesis imperfecta: comparison of molecular def

38 Ornoy A, Kim OJ. Scanning electron microscopy finding in osteogenesis imperfecta lethalis. Isr $\mathcal{F}$ Med Sci 1977;13: 26-32.

39 Brenner RE, Vetter U, Nerlich A, Wörsdorfer O, Telle WM, Müller PK. Osteogenesis imperfecta: insufficient collagen synthesis in early childhood as evidenced by collagen synthesis in early childhood as evidenced by analysis of compact bone and
Clin Invest 1989;19:159-66.

40 Niyibizi C, Bonadio J, Byers PH, Eyre DR. Incorporation of type I collagen molecules that contain a mutant $\alpha 2(\mathrm{I})$ chain (Gly ${ }^{50} \rightarrow$ Asp) into bone matrix in a lethal case of osteogenesis imperfecta. $\mathcal{F}$ Biol Chem 1992;267:23108-12.

41 Vetter U, Fisher LW, Mintz KP, et al. Osteogenesis imperfecta: changes in noncollagenous proteins in bone. $f$ Bone Mineral Res 1991;6:501-5.

42 Stöss H, Freisinger P. Collagen fibrils of osteoid in osteogenesis imperfecta: morphometrical analysis of the fibril diameter. Am f Med Genet 1993;45:257.

43 Vetter U, Eanes ED, Kopp JB, Termine JD, Robey PG Changes in apatite crystal size in bones of patients with osteogenesis imperfecta. Calc Tiss Int 1991;49:248-50.

44 Khillan JS, Olsen AS, Kontusaari S, Sokolov B, Prockop DJ. Transgenic mice that express a mini-gene version of the human gene for type I procollagen (COL1A1) develop the human gene for type I procollagen (COL1A1) develop a phenotype resembling a lethal form of
imperfecta. $₹$ Biol Chem 1991;266:23373-9.

45 Cole WG, Campbell PE, Rogers JG, Bateman JF. The clinical features of osteogenesis imperfecta resulting from a non-functional carboxyterminal proal(I) propeptide of type I procollagen and a severe deficiency of normal type I collagen in tissues. $\mathcal{f}$ Med Genet 1990;27:545-51.

46 Willing MC, Cohn DH, Byers PH. Frameshift mutation near the $3^{\prime}$ end of the COL1A1 gene of type I collagen predicts an elongated pro $\alpha 1$ (I) chain and results in osteogenesis imperfecta type I. $\mathcal{F}$ Clin Invest 1990;85:282-90.

47 Williams EM, Nicholls AC, Daw SCM, et al. Phenotypical features of a unique Irish family with severe autosoma recessive

48 Bonadio J, Saunders TL, Tsai E, et al. Transgenic mouse model of the mild dominant form of osteogenesis immodel of the mild dominant form of osteogenesis

49 Schnieke A, Harbers K, Jaenisch R. Embryonic lethal mutation in mice induced by retrovirus insertion into the $\alpha 1$ (I) collagen gene. Nature 1983;304:315-20.

50 Nicholls AC, Osse G, Schloon HG, et al. The clinica features of homozygous $\alpha 2(\mathrm{I})$ collagen deficient osteogenesis imperfecta. $\mathcal{F}$ Med Genet 1984;21:257-62.

51 Fertala A, Westerhausen A, Morris G, Rooney JE, Prockop DJ. Two cysteine substitutions in procollagen I: a glycine replacement near the $\mathrm{N}$-terminus of $\alpha 1$ (I) chain causes lethal osteogenesis imperfecta and a glycine replacement in the $\alpha 2(\mathrm{I})$ chain markedly destabilizes the triple helix. Biochem F 1993;289:195-9.

52 Rose NJ, Mackay K, Byers PH, Dalgleish R. A Gly859Ser substitution in the triple helical domain of the $\alpha 2$ chain of type I collagen resulting in osteogenesis imperfecta type

III in two unrelated individuals. Hum Mutat 1994;3:391-4 HH, Dalgleish K, De Paepe A, Steinmann B, Punnet HH, Dalgleish R. Three unrelated individuals with periical Gly502Ser substitutions in the $\alpha 2$-chain of type ical Gly502Ser substitutions in the $\alpha 2-c$

54 Pereira R, Khillan JS, Helminen HJ, Hume EL, Prockop DJ. Transgenic mice expressing a partially deleted gene for type I procollagen (COL1A1). A breeding line with a phenotype of spontaneous fractures and decreased bone collagen and mineral. $f$ Clin Invest 1993;91:709-16.

55 Cole WG, Patterson E, Bonadio J, Campbell PE, Fortun DW. The clinicopathological features of three babies with osteogenesis imperfecta resulting from the substitution of glycine by valine in the proal(I) chain of type I procollagen. $\mathcal{F}$ Med Genet 1992;29:112-8.

56 Constantine G, McCormack J, McHugo J, Fowlie A. Prenatal diagnosis of severe osteogenesis imperfecta. Prenat Diagn 1991;11:103-10.

57 Sharony R, Browne C, Lachman RS, Rimoin DL. Prenatal diagnosis of the skeletal dysplasias. Am $\mathcal{F}$ Obstet Gynecol 1993;169:668-75.

58 Morin LRM, Herlicoviez M, Loisel JC, Jacob B, Feuilly imperfecta in twin pregnancy. Clin Genet 1991;39:467-70. 